\title{
Morphological evolution from a rough to biphased surface on $\mathrm{TiO}_{2}(100)$
}

\author{
Tomoyuki Uehara, Mari Yoshihara, and Toshio Ogino \\ Yokohama National University \\ 79-5, Tokiwadai, Hodogaya, Yokohama 240-8501, Japan \\ Corresponding author: T. Ogino, togino@ynu.ac.jp , +81-45-339-4147
}

\begin{abstract}
We found that a rutile single-crystalline $\mathrm{TiO}_{2}(100)$ surface exhibits "biphased" structure in air that consists

of structurally and chemically different domains. We observed their structural evolution caused by thermal annealing at $900{ }^{\circ} \mathrm{C}$ by the contact mode of atomic force microscopy (AFM) and investigated their chemical properties by frictional mode AFM, which can be used to estimate local hydrophilicity of the surface. The two domains are distinguished by their morphological height and hydrophilicity, the latter of which is determined by the density of hydroxyl groups. The structural evolution and the final domain arrangement upon annealing are influenced by the initial atomic step arrangement before the annealing. We also demonstrated that a self-assembled monolayer of the silane-coupling agent is selectively formed only on the hydrophilic domains and that photochemical reaction using ultraviolet light eliminates the chemically characterized biphase feature.
\end{abstract}

Keywords: titanium oxide; surface; phase separation; atomic force microscopy; self-assembled monolayer 


\section{Introduction}

Titanium dioxide $\left(\mathrm{TiO}_{2}\right)$ is one of the most widely used metal oxides owing to its unique properties, such as photochemical reactivity and superhydrophilicity, and has been widely studied since photocatalytic activity for water decomposition was found in 1972 [1]. $\mathrm{TiO}_{2}$ surfaces exhibit strong oxidation power [2], and highly amphiphilic surfaces are generated by UV irradiation [3]. These properties have been applied to $\mathrm{TiO}_{2}$-coated glasses and tiles that exhibit self-cleaning, antifog and antifouling [4-5]. Since chemical properties such as water-dissociation and electronic properties such as work function are closely related to atomic structure of the surface [6-9], surface reconstructions have been intensively studied. $\mathrm{TiO}_{2}$ has some stable crystal phases, and the most stable one among them is rutile-type. The (110) face is the most stable surface of the rutile $\mathrm{TiO}_{2}$, and its atomic structure is well understood [10,11]. On the $\mathrm{TiO}_{2}(100)$ face, two kinds of reconstruction have been reported [12-16]: $(1 \times 1)$ and $(1 \times 3)$. The $(1 \times 1)$ surface is atomically flat and chemically stable, whereas the $(1 \times 3)$ surface, which is characterized by grooves running along [001] direction, was formed by ion-sputtering and subsequent thermal annealing [12]. In addition to surface reconstruction, atomic steps often play an important role in surface reactions. On vicinal $\mathrm{TiO}_{2}$ surfaces, a regularly ordered step-terrace structure appears by chemical treatment or annealing in air $[17,18]$. Since the $\mathrm{TiO}_{2}$ surface is not further oxidized, its atomic-level structure is generally kept unchanged in air or in an aqueous solution. For example, on $\mathrm{TiO}_{2}(100)$ surfaces, monolayer steps with a height of half of the unit cell $(0.23 \mathrm{~nm})$ form upon annealing in an oxygen flow at $700{ }^{\circ} \mathrm{C}$, whereas bunched steps appear when annealed at $800{ }^{\circ} \mathrm{C}[19]$. 
Another surface structure specific to metal oxide crystals is a chemical domain. We have already reported a chemically phase-separated surface, which we refer to as a biphased surface in this paper, on sapphire $\left(\mathrm{Al}_{2} \mathrm{O}_{3}\right)(0001)$ and (1-102) surfaces [20-23]. The notable feature of these surfaces is that domains with different hydrophilicity and surface charge, in addition to a difference in the geometry, coexist on one plane. We have also demonstrated that various protein molecules are selectively adsorbed to either or both of the domains on the biphased surface depending on the molecule isoelectric point and structure [22]. Therefore, application of the biphased surface to biodevices is promising because nanoscaled domain structure is spontaneously assembled, and because separation of protein species can be achieved by a simple process. The biphased surfaces are useful for any study that examines the effects of substrate surface. Since two different domains coexist on a nano- or micro-scaled surface area, any different phenomenon appearing on the different domains can be unambiguously attributed to the difference in the surface geometry and/or surface chemistry $[22,24]$ because the other experimental conditions are exactly same.

In this paper, we report on a new biphased surface on rutile $\mathrm{TiO}_{2}(100)$ surfaces formed by thermal annealing. Emphasis is on morphological evolutions from step-ordered or step-disordered surfaces to the biphased surface. We observed the phase-separation process by the contact mode of atomic force microscopy (AFM) and detected the hydrophilicity of the formed domains by the frictional mode of AFM. The differences in chemical termination of the phase-separated domains and their photochemical reaction under irradiation of ultraviolet (UV) light were investigated by observing formation behavior of self-assembled monolayers (SAMs). 


\section{Experimental Procedure}

The Verneuil-grown rutile $\mathrm{TiO}_{2}(100)$ wafers with a miscut of about $0.5^{\circ}$ toward the [011] orientation were purchased from Furuuchi Chemical Corporation. They were nominally non-doped with purity of 99.99\% and used without additional purification. The wafers were divided into small chips and sonicated in acetone, ethanol and deionized water $\left(>18.2 \mathrm{M} \Omega \mathrm{cm}^{-1}\right)$ for 5 min each to clean the surface. To prepare ordered step-terrace structure, some chips were immersed in a $\mathrm{H}_{2} \mathrm{SO}_{4}$ and $\mathrm{H}_{2} \mathrm{O}_{2}$ mixed solution (volume ratio of 3:1) for $10 \mathrm{~s}$, etched by a $10 \% \mathrm{HF}$ solution for $10 \mathrm{~min}$ and annealed in an $\mathrm{O}_{2}$ flow $\left(1.0 \mathrm{~L} \mathrm{~min}^{-1}\right)$ at $700{ }^{\circ} \mathrm{C}$ for $1 \mathrm{~h}$ [19]. We refer to the chips with and without the above-mentioned chemical and thermal treatments as step-arranged and pristine samples, respectively. To form biphased surfaces, both the pristine and step-arranged samples were annealed in air at $900{ }^{\circ} \mathrm{C}$ for $3 \mathrm{~h}$ or $5 \mathrm{~h}$. Upon this annealing, surface atomic structure is reconstructed and morphological evolution to the biphased surface occurs. Surface topography and local hydrophilicity distribution were simultaneously obtained by the contact mode and the frictional force mode of AFM, respectively. Since the frictional force on atomically flat surfaces is generated mainly by the meniscus between the tip and the sample surface, its distribution directly display local hydrophilicity on the surface. This was clearly demonstrated in a sample with a graphene film on a sapphire surface where graphene exhibits strong hydrophobicity and sapphire surface hydrophilicity [25].We used cantilevers with spring constants of $0.09 \mathrm{~N} / \mathrm{m}$ for vertical bending and $0.10 \mathrm{~N} / \mathrm{m}$ for lateral torsion, and measurements were performed in air without humidity control. Crystal orientation in the $\mathrm{TiO}_{2}$ surface was determined by 
electron backscatter diffraction (EBSD).

To more directly demonstrate a difference in surface termination states in the domains on the biphased $\mathrm{TiO}_{2}(100)$ surfaces, we used silane-coupling agent as a SAM because its formation is sensitive to the density of surface-terminating hydroxyl $(\mathrm{OH})$ group, which often is the major origin of the surface hydrophilicity of metal oxides. Octadecyltrichlorosilane (OTS, $\left.\mathrm{CH}_{3}\left(\mathrm{CH}_{2}\right)_{17} \mathrm{SiCl}_{3}\right)$ is one of the silane coupling agents and form a monolayer in the hydrophilic areas through a reaction between the $\mathrm{SiCl}_{3}$ group of an OTS molecule and the $\mathrm{OH}$ group of the $\mathrm{TiO}_{2}$ surface [26]. Therefore, the surface termination states can be unambiguously determined by observation of OTS-SAM formation. In the OTS-SAM deposition, the biphased $\mathrm{TiO}_{2}(100)$ wafers were sonicated in acetone, ethanol and deionized water for 5 minutes each. Some of the cleaned surfaces were irradiated with UV light (Meiwafosis PC-450) for 1h to make the surface superhydrophilic. An OTS solution with $0.25 \mathrm{ml}$ OTS and $40 \mathrm{ml}$ dehydrated toluene was prepared
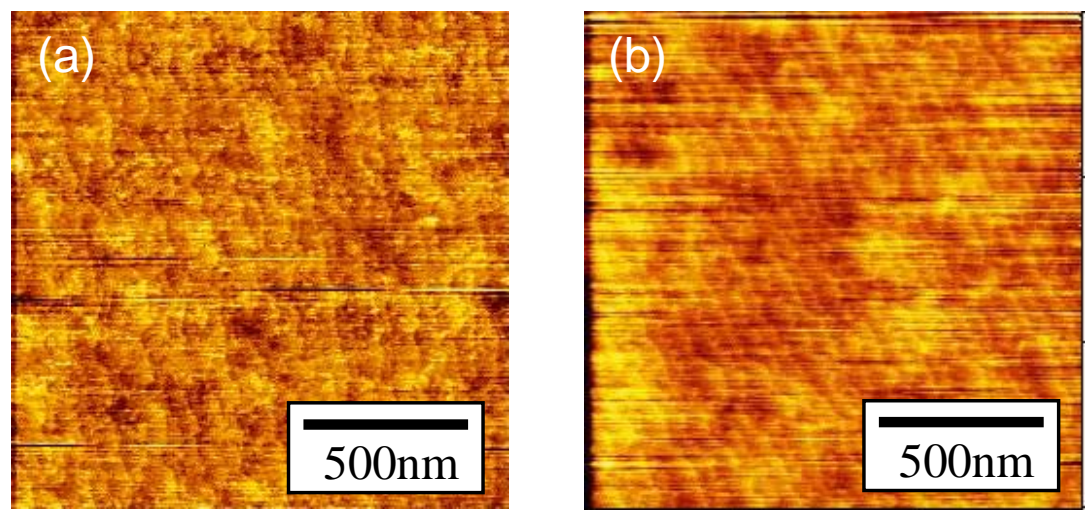

Fig. 1. AFM topographies of $\mathrm{TiO}_{2}(100)$ surfaces without thermal annealing: (a) a pristine surface and (b) a step-arranged one by chemical and thermal process. 
in a glove box at the humidity below $20 \%$. The UV-irradiated and non-irradiated samples were immersed in the OTS solution for 5 minutes. After deposition of OTS SAMs, the samples were sonicated in toluene, acetone, ethanol and deionized water for 5 min each to remove the physisorbed OTS molecules. The sample surfaces after OTS deposition were observed by the contact mode of AFM.

\section{Results and discussion}

Fig. 1a,b shows an AFM topography of a pristine $\mathrm{TiO}_{2}(100)$ surface and that of a step-arranged one,

respectively. In Fig. 1b, straight steps are uniformly distributed and their heights are from 0.2 to $0.3 \mathrm{~nm}$.
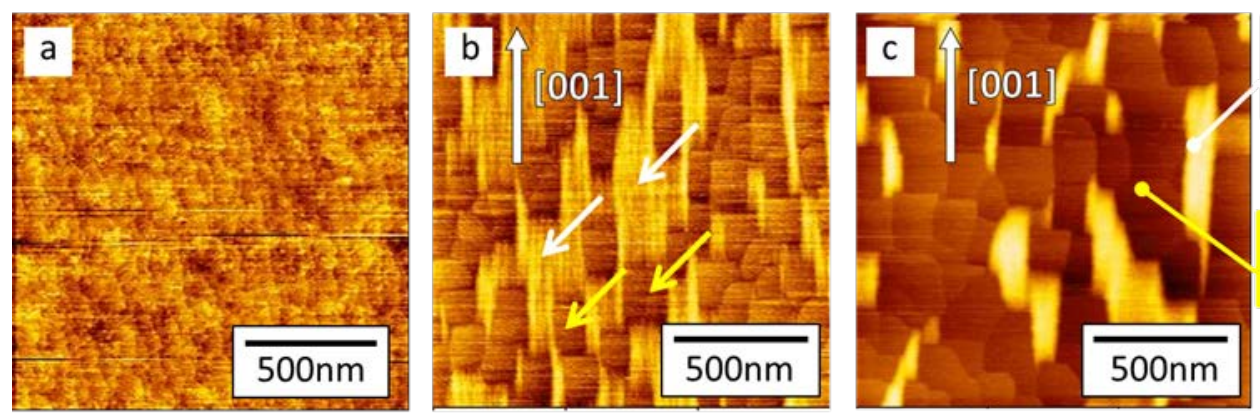

high domain
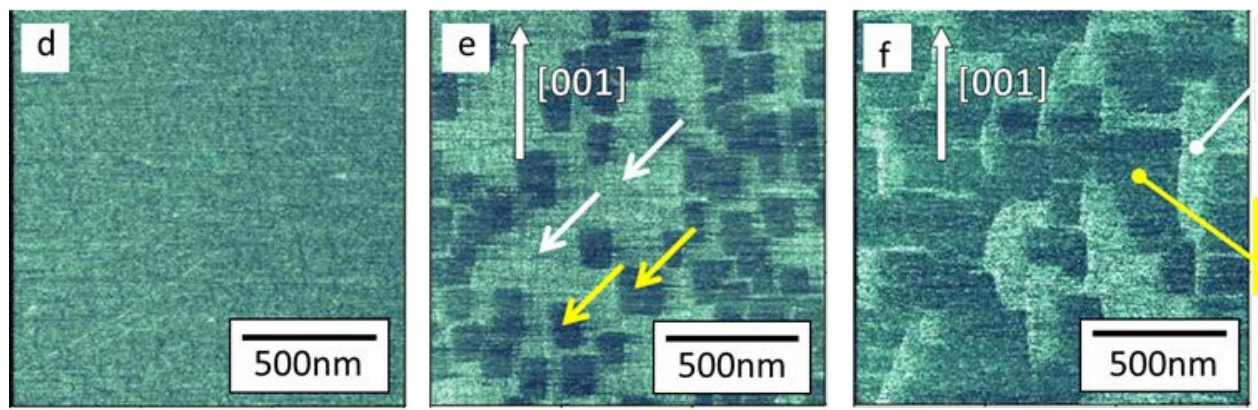

hydrophilic domain

hydrophobic domain

Fig. 2. AFM topographies (a-c) and frictional images (d-f) that demonstrate biphased surface formation process from a pristine $\mathrm{TiO}_{2}(100)$ surface: (a,d) without annealing (b,e) after annealing at $900{ }^{\circ} \mathrm{C}$ for $3 \mathrm{~h}$, and (c,f) after annealing at $900{ }^{\circ} \mathrm{C}$ for $5 \mathrm{~h}$. 
Theoretical step height for the (100) surface is $0.25 \mathrm{~nm}$, and that of $0.27 \mathrm{~nm}$ was reported using AFM [17].

Therefore, monolayer steps are formed on the surface shown in Fig. 1b.

Fig. 2 shows AFM topographies (a-c) and frictional images (d-f) that demonstrate formation process of a biphased surface from the pristine $\mathrm{TiO}_{2}$ surface. Before annealing, the surface is rough, as shown in Fig. 2a, but its hydrophilicity is uniform, as shown in Fig. 2d. After 3h annealing, the surface is divided into high and low terraces, as denoted by white and yellow arrows in Fig. 2b, respectively. In the frictional image shown in Fig. 2e, the surface is also divided into bright and dark areas, as denoted by white and yellow arrows, which correspond to hydrophilic and hydrophobic domains, respectively. By comparing Fig. 2b and 2e, we can find that the higher-in-topography terraces are relatively hydrophilic and the lower-intopography ones hydrophobic, which we refer to as the hydrophilic and hydrophobic domains, respectively. This means that, upon thermal annealing, the $\mathrm{TiO}_{2}(100)$ surface is phase-separated into two domains that morphologically and chemically differ. The hydrophilic domains exhibit striped patterns elongated in the [001] direction whereas the low domains are relatively flat and rectangular. Fig. 2c,f shows topographies and frictional images of the sample annealed for $5 \mathrm{~h}$. The stripe pattern of the hydrophilic domains disappeared, and their area fraction on the whole surface decreased from $65 \%$ to $35 \%$. Ordered domain shapes, however, were not obtained. 
Fig. 3 shows AFM topographies (a-c) and frictional images (d-f) on the step-arranged sample upon

thermal annealing at $900{ }^{\circ} \mathrm{C}$. Before annealing, no domain structure was observed, as shown in Fig. 3a,d.

Afte annealingr for $3 \mathrm{~h}$, as shown in Fig. 3b,e, lower-in-topography domains (yellow arrows) with regularly rectangular shapes appeared, and their frictional image shown in Fig. 3e confirms that those rectangular domains correspond to hydrophobic ones. After annealing for 5 h, as shown in Figs. 3c,f, the phase-separation proceeded, and the area fraction of the hydrophobic domains on the whole surface increased from $14 \%$ to $38 \%$ though the hydrophilic ones (white arrows) remained predominat. Obviously, mechanism of the phase-separaion on the step-arraged surface is the same as that developed on the pristine surface. However, the area fraction of the hydrophilic domains in the step-arranged sample (62\%) is much
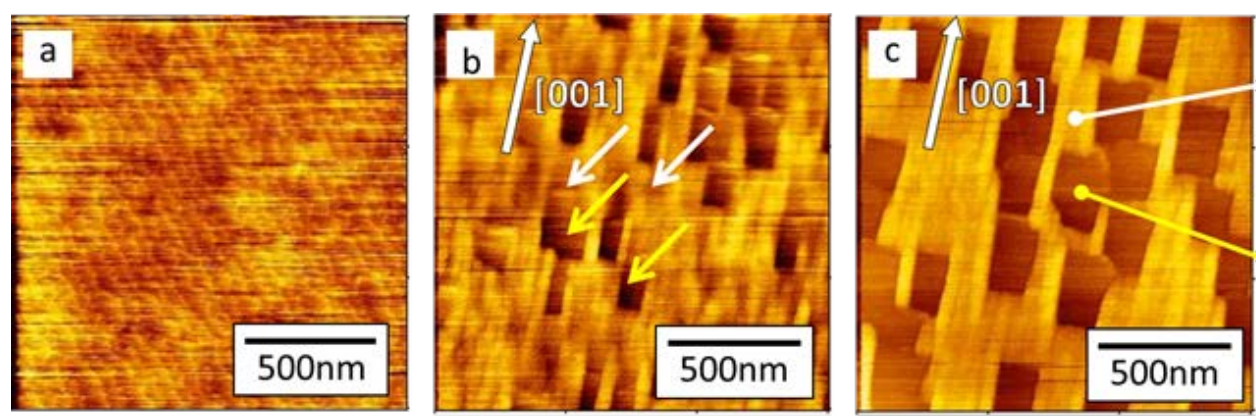

high domain
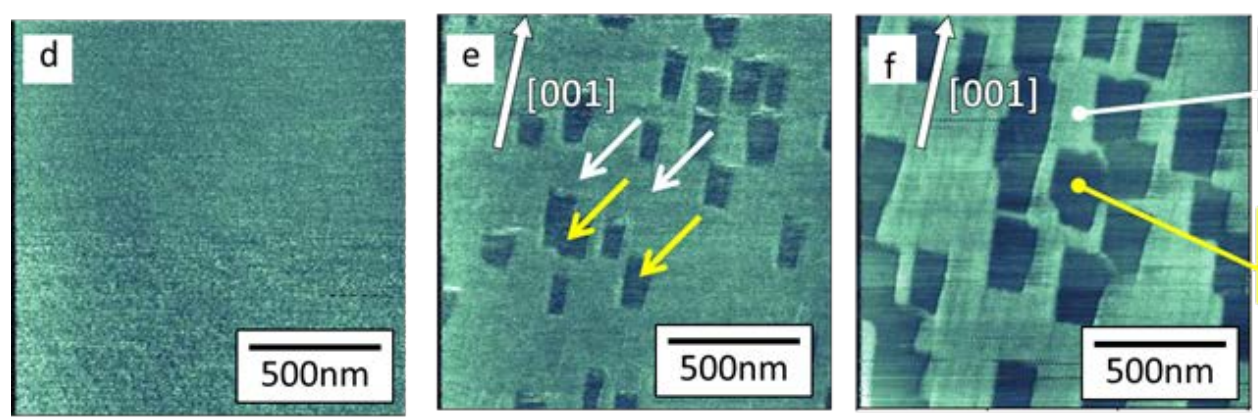

hydrophilic domain

hydrophobic domain

Fig. 3 AFM topographies (a-c) and frictional images (d-f) that demonstrate biphased surface formation process from a step-arranged $\mathrm{TiO}_{2}(100)$ surface: (a,d) without annealing (b,e) after annealing at $900{ }^{\circ} \mathrm{C}$ for $3 \mathrm{~h}$, and (c,f) after annealing at $900{ }^{\circ} \mathrm{C}$ for $5 \mathrm{~h}$. 
larger than that in the pristine sample (35\%) after 5h annealing. This result indicates that the advance step-ordering is effective to make a more regular biphased surface.

Fig. 4 shows cross-sectional views of the biphased surfaces formed from (a) the pristine and (b ) the step-arranged $\mathrm{TiO}_{2}$ (100) surfaces, and Fig. 4c,d their three-dimensional views. On the surface formed from the pristine sample shown in Fig. 4a, height of the hydrophilic domains with respect to the adjacent
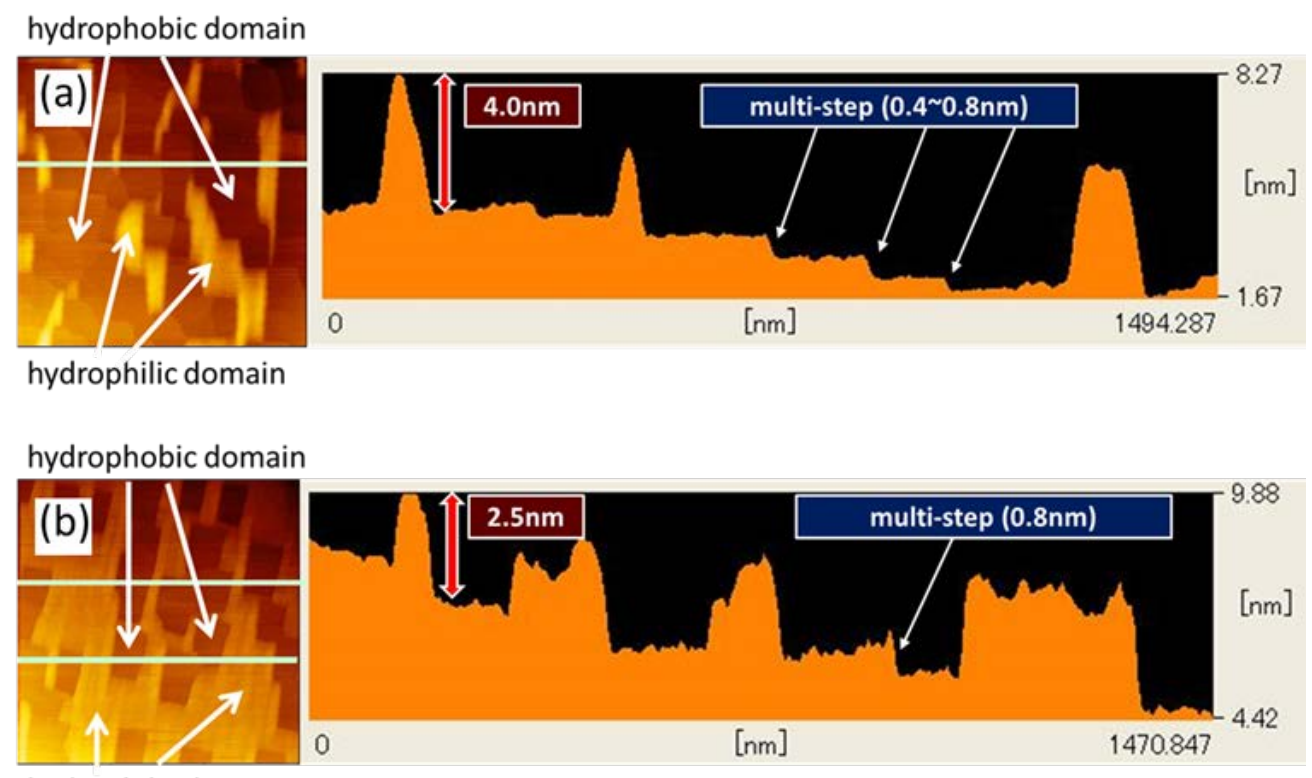

hydrophilic domain
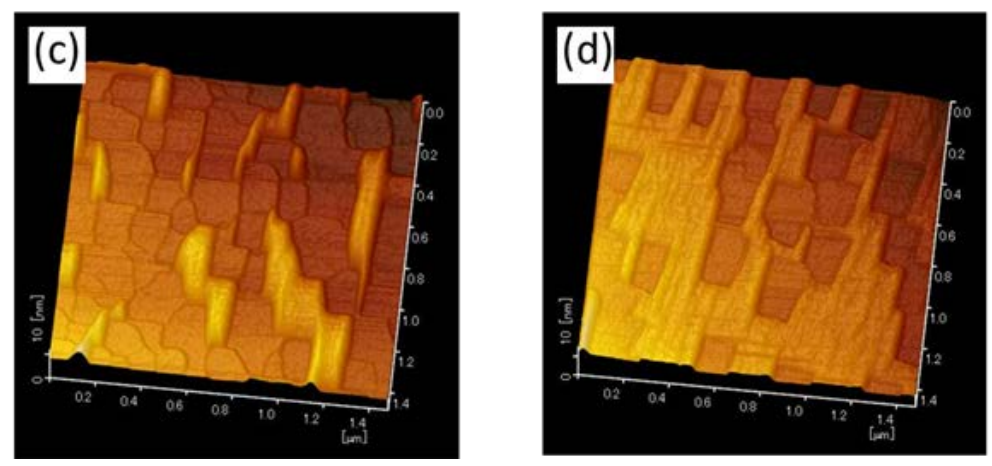

Fig. 4. Cross-sectional profiles of the biphased $\mathrm{TiO}_{2}(100)$ surfaces observed by AFM: (a) a biphase formed from the pristine surface and (b) that from the step-arranged surface. (c) and (d) are their 3D profiles. The image sizes are $1.5 \times 1.5 \mu \mathrm{m}$. 
hydrophobic domains is 3-4 nm, and the hydrophobic domains contain multi-steps whose height is $0.4-0.8$ nm. On the surface formed from the step-arranged sample shown in Fig. 4(b), the hydrophilic domains are about $2.5 \mathrm{~nm}$ high with respect to the hydrophobic domains and exhibit well-defined flat-terrace and multi-step structure.

From the AFM topography shown in Fig. 2b and the crystal orientation obtained by EBSD, it was found that the hydrophilic domains grow in the [001] direction in the early stage of phase-separation. The [001] is the elongated direction of the (110) microfacets that appear on the $1 \times 3$ reconstructed surface $[12,13,27,28]$. Generally, metal oxide surfaces can also be reconstructed upon annealing in air $[20,23]$ or in oxygen atmosphere [29]. Although the surface reconstruction in air is different from that in ultrahigh vacuum, the [001] is a specific direction that both of the $1 \times 3$ reconstruction and the present biphase have in common. Domain pattern of the biphased surface formed from the step-arranged surface differs from that from the pristine surface. This is probably owing to a difference in the step density because step edges are often starting points of surface reconstruction and because nucleation of the hydrophobic and/or hydrophilic domains more randomly occurs on the pristine surface than the step-arranged one. 


\subsection{OTS formation on biphased surfaces}

From AFM frictional images, biphased $\mathrm{TiO}_{2}(100)$ surface exhibits two domains with different

hydrophilicity, which depends on the density of OH groups. Fig. 5a,d shows AFM topography and

frictional image of a biphased $\mathrm{TiO}_{2}(100)$ surface obtained by the same process as that for Fig. 3. In Fig.

5a,d, the hydrophobic domains are higher in topography with respect to the hydrophilic domains, which is
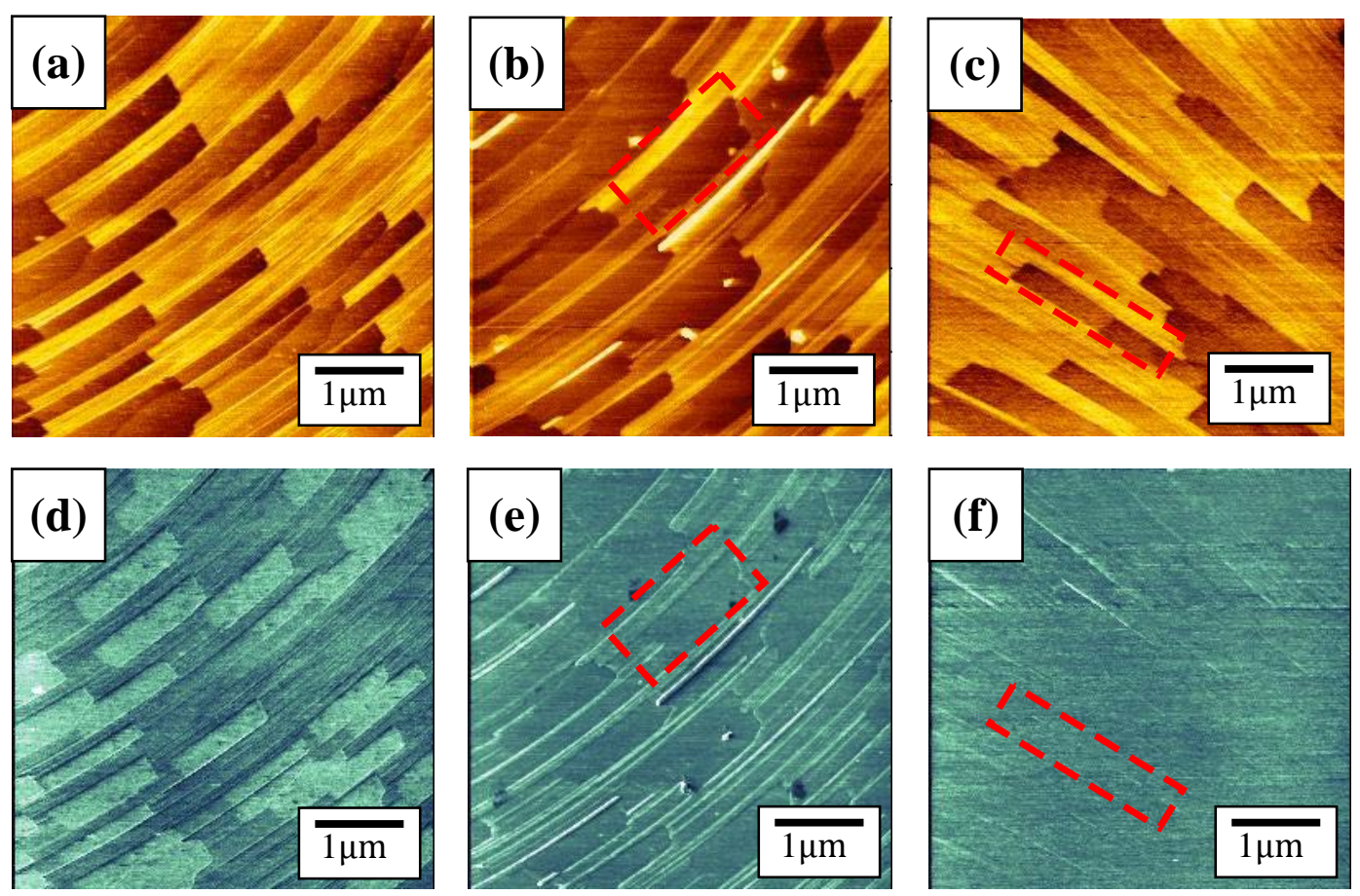

Fig. 5. AFM topographies (a-c) and frictional images (d-f) of $\mathrm{TiO}_{2}(100)$ surfaces that demonstrate deposition selectivity of an OTS-SAM: (a,d) an original biphased surface without UV irradiation nor OTS-SAM deposition, (b,e) surface after an OTS-SAM was deposited on the non-UV-irradiated biphased surface and (c,f) surface after an OTS-SAM was deposited on the UV-irradiated biphased surface. 
opposite to the result shown in Fig. 3. However, we often observed the height reversal between the hydrophilic and hydrophobic domains, and the difference in hydrophilicity is more essential than the difference in height. The domain pattern in Fig. 5a,d is also different from that in Fig. 3. The morphological difference between Figs. 3 and 5 is probably attributed to difference in the parent crystals, which were purchased in different years. Since another $\mathrm{TiO}_{2}$ wafer was used in Fig. 5, the miscut and its orientation direction also differ. Therefore, the formed pattern in this experiment was not exactly same as that in Fig. 3, but its feature as a biphased surface is the same as the sample used in Fig. 3. Fig. 5b,e shows AFM topography and frictional image of a surface where an OTS-SAM was deposited on the biphased surface without UV irradiation. In Fig. 5b,e, the originally hydrophilic domains, for example the domain indicated by a red rectangle, exhibits larger hydrophobicity than the originally hydrophobic domains. This hydrophilicity reversal by the OTS deposition is interpreted as preferential deposition of OTS-SAM on the OH-group-rich, originally hydrophilic domains, as shown in Fig. 6a. OTS molecules are chemically bonded with a $\mathrm{TiO}_{2}$ surface through reaction between the $\mathrm{Si}^{-C_{3}}$ head groups of OTS molecules and OH-groups of the $\mathrm{TiO}_{2}$ surface. Therefore, OTS molecule density bonded with $\mathrm{TiO}_{2}$ surface is higher on the area with higher OH-group density. Another head group of an OTS molecule opposite to chemical bond with the $\mathrm{TiO}_{2}$ surface is $\mathrm{CH}_{3}$, which is a nonpolar group. As a result, the hydrophilic domains, which was OH-group-rich areas, are converted to hydrophobic domains by selective formation of a high density OTS-SAM. 


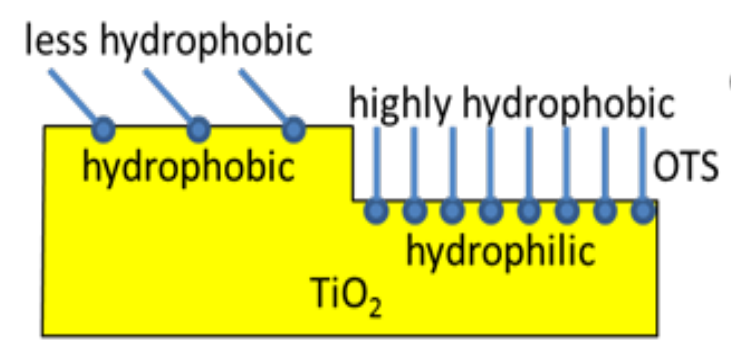

\section{highly hydrophobic}

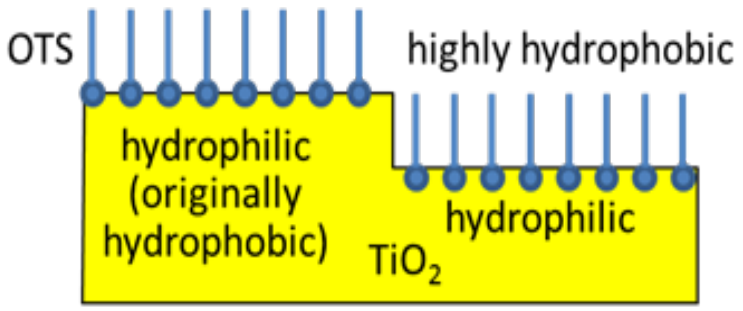

Fig. 6. Model of biphased $\mathrm{TiO}_{2}$ surfaces after OTS-SAM deposition: (a) non-UVirradiated surface and (b) UV-irradiated one.

Fig. 5c,f shows AFM topography and frictional image of a surface where an OTS-SAM was deposited on the UV-irradiated biphased surface, where no difference in hydrophilicity was observed, as indicated by a red rectangle. This uniform hydrophilicity indicates that the OTS-SAM was uniformly deposited on the UV-irradiated surface. UV-irradiation makes $\mathrm{TiO}_{2}$ surfaces superhydrophilic. The result shown in Fig. $5 f$ indicates that the UV irradiation destroy the phase-separated domain structure.

Finally, we discuss feasibility of the biphased surfaces for biotechnology. We have already demonstrated that various biomolecules can be separated on biphase sapphire (0001) surfaces by utilizing difference in chemical properties of the domains [21,22]. Selective adsorption of biomolecules to solid surfaces can be realized by other several methods: for example, use of peptide that recognizes a specific metal surface such as Ti-binding protein [30], surface modification using self-assembled monolayers [31] or use of graphene films attached to SAM surfaces [32]. However, these methods have drawbacks such as complicated fabrication process of the peptide, use of unstable monolayer surfaces or poor scalability, respectively. Since the biphased surfaces are stable and can be prepared by simple thermal annealing of the substrate. 
Moreover, they can be used repeatedly by recovering the clean surface using only chemical process.

Therefore, biphased surfaces are useful for biodevices that separate and detect biomolecules.

\section{Conclusion}

We have found that biphased structure appears on $\mathrm{TiO}_{2}(100)$ surfaces upon thermal annealing and observed its morphological evolution by AFM. The biphased surfaces consist of hydrophilic and hydrophobic domains that are accompanied with height difference in geometry. We also found that pre-arrangement of step-terrace structure is effective to form more well-defined biphased surface because morphological evolution to the biphase is initiated at step edges. By selective bindings of OTS films, we have confirmed that the difference in hydrophilicity is attributed to difference in OH-group density. After UV irradiation, an OTS-SAM uniformly forms on the surface that was initially phase-separated, indicating that both domains, at least the hydrophobic domains, are converted to hydrophilic surfaces.

\section{Acknowledgement}

This work was supported by Grant-in-Aid for Scientific Research from the Ministry of Education, Culture, Sports, Science and Technology (24360013).

\section{References}

[1] A. Fujishima, K. Honda, Electrochemical photolysis of water at a semiconductor electrode, Nature, 238 (1972) 37-38. 
[2] I. Sopyan, M. Watanabe, S. Murasawa, K. Hashimoto, A. Fujishima, An efficient $\mathrm{TiO}_{2}$ thin-film photocatalyst: photocatalytic properties in gas-phase acetaldehyde degradation, J. Photochem. Photobiol. A Chem. 98 (1996) 79-86.

[3] R. Wang, K. Hashimoto, A. Fujishima, M. Chikuni, E. Kojima, A. Kitamura, M. Shimohigoshi, T. Watanabe, Photogeneration of highly amphiphilic $\mathrm{TiO}_{2}$ surfaces, Adv. Mater. 10 (1998) 135-138.

[4] T. Watanabe, A. Nakajima, R. Wang, M. Minabe, S. Koizumi, A. Fujishima, K. Hashimoto. Photocatalytic activity and photoinduced hydrophilicity of titanium dioxide coated glass, Thin Solid Films, 351 (1999), 260-263.

[5] T. Noguchi, A. Fujishima, P. Sawunyama, K. Hashimoto, Photocatalytic degradation of gaseous formaldehyde using $\mathrm{TiO}_{2}$ film, Environ. Sci. Technol. 32 (1998) 3831-3833.

[6] M. A. Henderson, Structural sensitivity in the dissociation of water on $\mathrm{TiO}_{2}$ single-crystal surfaces, Langmuir, 12 (1996) 5093-5098.

[7] R. Nakamura, T. Okamura, N. Ohashi, A. Imanishi, Y. Nakato, Molecular mechanisms of photoinduced oxygen evolution, PL emission, and surface roughening at atomically smooth (110) and (100) n- $\mathrm{TiO}_{2}$ (Rutile) surfaces in aqueous acidic solutions, J. Am. Chem. Soc. 127 (2005) 12975-12983.

[8] A. Imanishi, T. Okamura, N. Ohashi, R Nakamura, Y. Nakato, Mechanism of water photooxidation reaction at atomically flat $\mathrm{TiO}_{2}$ (rutile) (110) and (100) surfaces: dependence on solution pH, J. Am. Chem. Soc. 129 (2007) 11569-11578.

[9] A. Imanishi, E. Tsuji, Y. Nakato, Dependence of the work function of $\mathrm{TiO}_{2}$ (rutile) on crystal faces, studied by a scanning Auger microprobe, J. Phys. Chem. C, 111 (2007) 2128-2132.

[10] U. Diebold, The surface science of titanium dioxide, Surf. Sci. Rep. 48 (2003) 53-229.

[11] C. L. Pang, R. Lindsay, G. Thornton, Structure of clean and adsorbate-covered single-crystal rutile $\mathrm{TiO}_{2}$ surfaces, Chem. Rev. 113 (2013) 3887-3948.

[12] P. W. Murray, F. M. Leibsle, H. J. Fisher, C. F. J. Flipse, C. A. Muryn, G. Thornton, Observation of ordered oxygen vacancies on $\mathrm{TiO}_{2}(100) 1 \times 3$ using scanning tunneling microscopy and spectroscopy, Phys. Rev. B, 46 (1992) 12877-12879. 
[13] H. Zajons, H. L. Meyerheim, T. Gloege, W. Morits, D. Wolf, Surface X-ray structure analysis of the $\mathrm{TiO}_{2}(100)-(1 \times 3)$ reconstruction, Surf. Sci. 398 (1998) 369-378.

[14] E. Landree, L. D. Marks, P. Zshack, C. J. Gilmore, Structure of the $\mathrm{TiO}_{2-x}(100)-1 \times 3$ surface by direct methods, Surf. Sci. 408 (1998) 300-309.

[15] H. Raza, C. L. Pang, S. A. Haycock, G. Thornton, Evidence of discrete bond breaking steps in the $1 \times 1$ to $1 \times 3$ phase transition of $\mathrm{TiO}_{2}(100)$, Phys. Rev. Lett. 82 (1999) 5265-5268

[16] P. J. D. Lindan, N. M. Harrison, The structure of the reduced rutile $\mathrm{TiO}_{2}(100) 1 \times 3$ reconstruction, Surf. Sci. 479 (2001) L375-381.

[17] R. Nakamura, N. Ohashi, A. Imanishi, T. Osawa, Y. Matsumoto, H. Koinuma, Y. Nakato, Crystal-face dependences of surface band edges and hole reactivity, revealed by preparation of essentially atomically smooth and stable (110) and (100) n- $\mathrm{TiO}_{2}$ (rutile) surfaces, J. Phys. Chem. B 109 (2005) 1648-1651.

[18] Y. Yamamoto, K. Nakajima, T. Ohsawa, Y. Matsumoto, H. Koinuma, Preparation of atomically smooth $\mathrm{TiO}_{2}$ single crystal surfaces and their photochemical property, Jpn. J. Appl. Phys. 44 (2005) L511-L514.

[19] R. Tero, T. Ujihara, T. Urisu, Lipid bilayer membrane with atomic step structure: supported bilayer on step-and-terrace $\mathrm{TiO}_{2}(100)$ surface, Langmuir 24 (2008) 11567-11576.

[20] T. Isono, T. Ikeda, R. Aoki, K. Yamazaki, T. Ogino, Structural- and chemical-phase-separation on single crystalline sapphire (0001) surfaces, Surf. Sci. 604 (2010) 2055-2063.

[21] T. Isono, T. Ikeda, T. Ogino, Evolution of supported planar lipid bilayers on step-controlled sapphire surfaces, Langmuir 26 (2010) 9607-9611.

[22] K. Yamazaki, T. Ikeda, T. Isono, T. Ogino, Selective adsorption of protein molecules on phase-separated sapphire surfaces, J. Colloid Interface Sci. 361 (2011) 64-70.

[23] H. Komurasaki, T. Isono, T. Tsukamoto, T. Ogino, Evolution of step morphology on vicinal sapphire (1-102) surfaces accompanied with self-assembly of comb-shaped chemical domains, Appl. Surf. Sci. 258 (2012) 5666-5671.

[24] K. Saito, T. Ogino, Direct growth of graphene films on sapphire (0001) and (11-20) surfaces by self-catalytic chemical vapor deposition, J. Phys. Chem. C 118 (2014) 5523-5529. 
[25] T. Tsukamoto, T. Ogino, Morphology of graphene on step-controlled sapphire surfaces, Appl. Phys. Express 2 (2009) 075502 (1-3).

[26] A. Ulman, Formation and structure of self-assembled monolayers, Chem. Rev. 96 (1996) 1533-1554.

[27] H. Raza, C. L. Pang, S. A. Haycock, G. Thornton, Non-contact atomic force microscopy imaging of $\mathrm{TiO}_{2}(100)$ surfaces, Appl. Surf. Sci. 140 (1999) 271-275.

[28] N. Sawai, K. Yamasue, Y. Cho, Atomic scale imaging of $\mathrm{TiO}_{2}(100)$ reconstructed surfaces by noncontact scanning nonlinear dielectric microscopy, Jpn. J. Appl. Phys. 51 (2012) 121801(1-7).

[29] P. Stone, R. A. Bennett, M. Bowker, Reactive re-oxidation of reduced $\mathrm{TiO}_{2}(110)$ surfaces demonstrated by high temperature STM movies, New J. Phys. 1 (1999) 1.1-1.12.

[30] K. Sano, K. Shiba, A hexapeptide motif that electrostatically binds to the surface of titanium, J. Am. Chem.Soc. 125 (2003) 14234-14235.

[31] Y. Lin, J. Wang, L-J Wan, X-H. Fang, Study of fibrinogen adsorption on self-assembled monolayers on $\mathrm{Au}(111)$ by atomic force microscopy, Ultramicroscopy 105 (2005) 129-136.

[32] Y. Kamiya, K. Yamazaki, T. Ogino, Protein adsorption to graphene surfaces controlled by chemical modification of the substrate surfaces, J. Colloid Interface Sci. 431 (2014) 77-81.

Figure captions

Fig. 1. AFM topographies of $\mathrm{TiO}_{2}(100)$ surfaces without thermal annealing: (a) a pristine surface and (b) a step-arranged one by chemical and thermal process.

Fig. 2. AFM topographies (a-c) and frictional images (d-f) that demonstrate biphased surface formation process from a pristine $\mathrm{TiO}_{2}(100)$ surface: (a,d) without annealing (b,e) after annealing at $900{ }^{\circ} \mathrm{C}$ for $3 \mathrm{~h}$, and (c,f) after annealing at $900{ }^{\circ} \mathrm{C}$ for $5 \mathrm{~h}$. 
Fig. 3 AFM topographies (a-c) and frictional images (d-f) that demonstrate biphased surface formation process from a step-arranged $\mathrm{TiO}_{2}(100)$ surface: $(\mathrm{a}, \mathrm{d})$ without annealing (b,e) after annealing at $900{ }^{\circ} \mathrm{C}$ for 3h, and (c,f) after annealing at $900{ }^{\circ} \mathrm{C}$ for $5 \mathrm{~h}$.

Fig. 4. Cross-sectional profiles of the biphased $\mathrm{TiO}_{2}(100)$ surfaces observed by AFM: (a) a biphase formed from the pristine surface and (b) that from the step-arranged surface. (c) and (d) are their 3D profiles. The image sizes are $1.5 \times 1.5 \mu \mathrm{m}$.

Fig. 5. AFM topographies (a-c) and frictional images (d-f) of $\mathrm{TiO}_{2}(100)$ surfaces that demonstrate deposition selectivity of an OTS-SAM: (a,d) an original biphased surface without UV irradiation nor OTS-SAM deposition, (b,e) surface after an OTS-SAM was deposited on the non-UV-irradiated biphased surface and (c,f) surface after an OTS-SAM was deposited on the UV-irradiated biphased surface.

Fig. 6. Model of biphased $\mathrm{TiO}_{2}$ surfaces after OTS-SAM deposition: (a) non-UV- irradiated surface and (b) UV-irradiated one. 